\title{
Effects of Particle-Wall Interactions on the Thermodynamic Behavior of Gases at the Nano Scale
}

\author{
C. Firat $^{1}$, A. Sisman $* *^{2}$, Z.F. Ozturk ${ }^{3}$ \\ Istanbul Technical University, Energy Institute \\ E-mails: ${ }^{1}$ firat@energy.itu.edu.tr, ${ }^{2}$ sismanal@itu.edu.tr, ${ }^{3}$ ozturkzeh@itu.edu.tr
}

\begin{abstract}
The thermodynamic behavior of gases confined in nano structures is considerably different than those in macro ones due to the effects of both particle-wall interactions and the wave character of particles. The homogeneous density distribution of a gas at thermodynamic equilibrium is disturbed by these effects. Because of particle-wall interactions, the local density of a gas changes drastically near the domain boundaries. Also, the wave character of the particles causes an inhomogeneous density distribution, especially near the boundaries. Consequently, the apparent density (number of particles over the domain volume) is different than the real one. All the densitydependent thermodynamic properties are affected by the inhomogeneity in the density distribution. Therefore, it is important to consider these effects on local density to analyze the thermodynamic behaviors of gases confined in nano structures. The detailed analysis of these effects on local density also gives a base of knowledge for the experimental verification of quantum size effects on local density due to the wave character of particles. In this study, the density distributions of classical (Maxwellian) and quantum (both Fermi and Bose) gases are calculated and investigated by considering both particle-wall interactions and quantum size effects. The results can be used for experimental verification of quantum size effects on gas density as well as the modeling of nano heat engines.
\end{abstract}

Keywords: Quantum size effects; density distribution; Lennard-Jones potential; thermodynamic properties.

\section{Introduction}

Today, especially in parallel to progress in semiconductor technology, the development of nanotechnology makes the mechanical systems and structures at the micro/nano scale possible. Mechanical structures at the micro/nano scale such as gas turbines, pumps, mixers, heat exchangers, valves, etc., bring the following topics into consideration: how do the thermodynamic properties of gases at the nano scale differ, how can they be modeled, how can one make use of these differences, and how new devices and technologies can be developed at this scale. In this perspective, the subject of quantum size effects (QSE) on the thermodynamics of gases at the micro/nano scale is a new research area and has many potential applications from the technologies of genetics, space, and energy to military ones (Kang, Hwang, Lee, \& Lee, 2004; Kang \& Hwang, 2004; Moriarty, 2001; Terrones, Kamalakaran, Seeger, \& Rühle, 2000; Cumings \& Zettl, 2000; Hoummady \& Fujita, 1999; Lijima \& Ichihashi, 1993; Lijima, 1991).

At the nano scale, the thermodynamic properties (TP) of gases differ from those at macro scales. One of the reasons for this difference is the QSE, which become important when the thermal de Broglie wavelength of particles $\left(\lambda_{T}\right)$ is not negligible in comparison with the characteristic length of the system ( $L=V / A, V$ volume, $A$ surface area). In such a case, the continuum approximation for the energy eigenvalues of particles becomes invalid and the discrete nature of energy eigenvalues causes QSE, which are noticeable at the nano scale. QSE make the thermodynamic state functions depend on the geometry (shape) and size of the system, and QSE causes some new and interesting behaviors, which are not observed at the macro scale. Some of them are anisotropic gas pressure, gas diffusion due to size and geometry differences, thermosize effects like thermoelectric effects and the disappearance of the additivity of extensive quantities (Molina, 1996; Gutierrez \& Yanez, 1997; Pathria, 1998; Dai \& Xie, 2003; Sisman \& Müller, 2004; Dai \& Xie, 2004; Sisman, 2004; Pang, Dai, $\&$ Xie, 2006).

The homogeneous density distribution of a gas at thermodynamic equilibrium is disturbed by the wave character of particles. All the density dependent thermodynamic properties are affected by the inhomogeneity in the density distribution (Sisman, Ozturk, \& Firat, 2007; Firat, Sisman \& Ozturk, 2010; Firat \& Sisman, 2009).

The density distribution is disturbed also by particlewall interactions. The interactions occurring between the gas particles and the particles of the walls cause local density changes near the boundaries and, therefore, another size effect appears besides the quantum size effects. The modeling of the change in local density due to particle-wall interactions as well as the wave character of particles allows one to distinguish these different effects on the density distribution from each other. Therefore, model results provide a basis for an experimental verification of quantum size effects on the thermodynamic properties of gases at the nano scale. In this study, for this purpose, the local density changes in both classical (Maxwellian) and quantum (Fermi and Bose) gases are calculated and examined by considering particle-wall interactions and QSE. To consider QSE, energy eigenvalues and eigenfunctions of particles are obtained by solving the Schrödinger equation. For different temperatures, local density changes due to only particle-wall interactions are

*This paper is an updated version of a paper published in the ECOS'10 proceedings. It is printed here with permission of the authors and organizers. 
compared to the density changes due to both QSE and particle-wall interactions.

The common function used for the interaction potential for particle-wall interactions is the Lennard-Jones, LJ (12-

6), potential (Hirschfelder, Curtiss \& Bird, 1954; Israelachvili, 1992). Therefore, in this study, the LJ (12-6) potential is used for the calculations. Here, the local density distribution of a gas (e.g., He) confined in a rectangular box with wall of, e.g., $\mathrm{Si}$, and having dimensions of $\mathrm{L}_{\mathrm{x}}, \mathrm{L}_{\mathrm{y}}$ and $\mathrm{L}_{\mathrm{z}}$ is investigated. The wall-gas interaction is represented by the LJ. In a rectangular domain, 3D density distribution is simply represented here by the production of $1 \mathrm{D}$ result for each direction. Therefore, in this study, a onedimensional problem is discussed. One-dimensional problems are not only simplified problems. In fact, in nano systems, often only one of the dimensions is at a nano scale and therefore, most of the systems can be examined by considering one-dimensional problem. On the other hand, 2D and 3D quantum simulations, which are beyond the scope of this work, are also needed to fully understand the complexity of nano-scale particle-wall interactions as well as the wave character of particles.

\section{Particle-wall interaction in a 10 domain}

In this section, density distributions of both classical (MB) and quantum (FD and BE) gases are investigated under a LJ (12-6) potential in the 1D and 2D domains.

\subsection{Lennard-Jones (12-6) interaction potential}

For a 1D confinement domain, the LJ (12-6) potential function can be written as follows (Hirschfelder, Curtiss \& Bird, 1954; Israelachvili, 1992):

$U(x)=4 \varepsilon_{L J}\left[\left(\frac{r_{0}}{x}\right)^{12}-\left(\frac{r_{0}}{x}\right)^{6}\right]+4 \varepsilon_{L J}\left[\left(\frac{r_{0}}{L-x}\right)^{12}-\left(\frac{r_{0}}{L-x}\right)^{6}\right]$

where the parameters $r_{0}$ and $\varepsilon_{L J}$ are the characteristic constants in unit length and energy, respectively, $L$ is the domain size and $x$ refers to the position in the domain.

Instead of using the numerical values of the parameters used in the calculations directly, the purpose there is to obtain generalized conclusions by using their dimensionless values. Thus all lengths are divided by the domain length, $L$ and all energies are divided by $k_{b} T$ to obtain the dimensionless values. In this case, the dimensionless $\mathrm{LJ}$ (12-6) potential can be written as follows:

$\tilde{U}(\tilde{x})=\frac{4}{\tilde{T}}\left[\left(\frac{\tilde{r}_{0}}{\tilde{x}}\right)^{12}-\left(\frac{\tilde{r}_{0}}{\tilde{x}}\right)^{6}\right]+\frac{4}{\tilde{T}}\left[\left(\frac{\tilde{r}_{0}}{1-\tilde{x}}\right)^{12}-\left(\frac{\tilde{r}_{0}}{1-\tilde{x}}\right)^{6}\right]$

In this expression, the dimensionless function and variables are defined as $\tilde{U}(\tilde{x})=U(\tilde{x}) / k_{b} T, \tilde{r}_{0}=r_{0} / L, \tilde{x}=x / L, \tilde{T}=T / T_{L J}$ with $T_{L J}=\varepsilon_{L J} / k_{b}$, and $k_{b}$ equals to the Boltzmann constant. The dimensionless interaction potential between the wall and gas atoms confined in a one-dimensional domain (for $L=L_{\mathrm{x}}=1 \mathrm{~nm}$ ) is given in Figure 1.

In this study, helium gas confined in a domain made of silicon is considered. The LJ parameters for the $\mathrm{He}-\mathrm{Si}$ interaction are $r_{0}=0.2855 \mathrm{~nm}, \varepsilon_{L J}=0.2413 \mathrm{~kJ} \mathrm{~mol}^{-1}$, and $T_{L J}=29 \mathrm{~K}$ (Nagy, Tunega \& Liska, 1996).

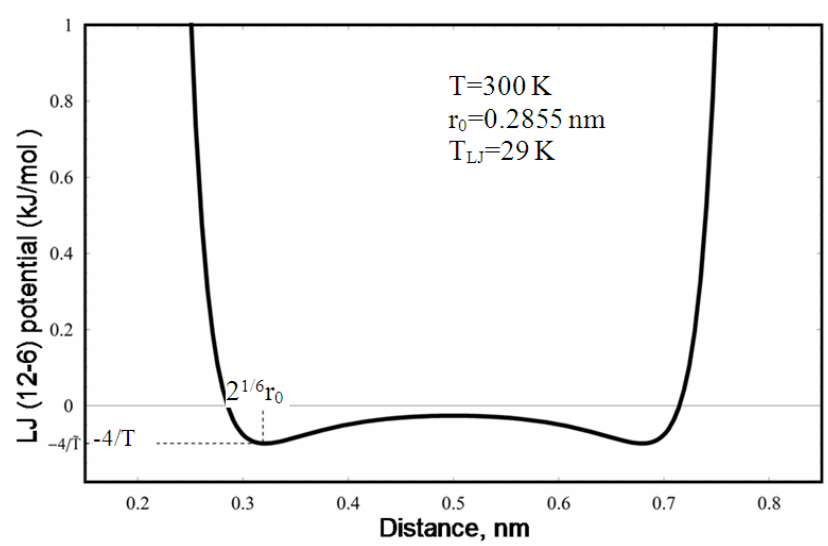

Figure 1. Dimensionless LJ(12-6) potential function for the He-Si interaction.

\subsection{Derivation of the density distribution equations}

For Fermi (FD) and Bose (BE) gases, the local density obtained by using dimensionless energy eigenvalues $\tilde{\varepsilon}_{r}$ and eigenfunctions $\psi_{r}(\tilde{x})$, which are the solutions of Schrödinger equation for the LJ potential, is expressed as follows (Sisman, Ozturk, \& Firat, 2007; Firat, Sisman \& Ozturk, 2010; Firat \& Sisman, 2009):

$$
\left(\begin{array}{l}
\mathrm{FD} \\
\mathrm{BE}
\end{array}\right) \quad n(\tilde{x})=\sum_{r} \frac{\left|\psi_{r}(\tilde{x})\right|^{2}}{\exp \left(-\Lambda+\tilde{\varepsilon}_{r}\right) \pm 1}
$$

where $\Lambda=\mu / k_{b} T$, is the dimensionless chemical potential in the absence of the LJ potential and is a (global) constant which is independent of the position.

The domain integral of Eq. (3) is equal to the total number of particles. Thus the apparent density (classical density) is written as follows:

$$
\begin{aligned}
\left(\begin{array}{c}
\mathrm{FD} \\
\mathrm{BE}
\end{array}\right) n_{c l} & =\frac{N}{L}=\frac{1}{\tilde{L}} \int_{0}^{\tilde{L}} n(\tilde{x}) d \tilde{x}=\frac{1}{\tilde{L}} \sum_{r} \int_{0}^{\tilde{L}} \frac{\left|\psi_{r}(\tilde{x})\right|^{2} d \tilde{x}}{\exp \left(-\Lambda+\tilde{\varepsilon}_{r}\right) \pm 1} \\
& =\frac{1}{\tilde{L}} \sum_{r} \frac{1}{\exp \left(-\Lambda+\tilde{\varepsilon}_{r}\right) \pm 1}
\end{aligned}
$$

Here, it should be noted that the integral of $\left|\psi_{r}(\tilde{x})\right|^{2}$ is equal to one. Remembering that all the lengths are divided by the domain size, the dimensionless density for both Fermi and Bose gases is written as follows $(\tilde{L}=1)$ :

$$
\left(\begin{array}{l}
\mathrm{FD} \\
\mathrm{BE}
\end{array}\right) \tilde{n}(\tilde{x})=\frac{n(\tilde{x})}{n_{c l}}=\frac{\sum_{r} \frac{\left|\tilde{\psi}_{r}(\tilde{x})\right|^{2}}{\sum_{r} \frac{1}{\exp \left(-\Lambda+\tilde{\varepsilon}_{r}\right) \pm 1}}}{\exp \left(-\Lambda+\tilde{\varepsilon}_{r}\right) \pm 1}
$$

In this equation, the eigenfunctions are also in dimensionless form. The dimensionless Schrödinger equation can be written as follows (Griffiths, 1995):

$$
-\frac{d^{2} \tilde{\psi}}{d \tilde{x}^{2}}+\left(\frac{\pi}{\alpha}\right)^{2} \tilde{U} \tilde{\psi}=\left(\frac{\pi}{\alpha}\right)^{2} \tilde{\varepsilon} \tilde{\psi}
$$

In this expression, $\tilde{\varepsilon}=\varepsilon / k_{b} T$ and $\alpha=L_{c} / L$ where $L_{c}=h /\left(2 \sqrt{2 m k_{b} T}\right)$ half of the most probable de Broglie wavelength and $m$ is the mass of a particle. 
For a potential function given by Eq. (2), Eq. (6) cannot be solved analytically. The eigenfunctions and eigenvalues can be obtained only by numerical methods. By using the numerical solution of the Schrödinger equation, the dimensionless local density distribution of a gas confined in a one dimensional domain is obtained.

In order to determine how much the local density distribution changes due to the existence of QSE, the distribution is calculated first by considering only the LJ potential and then by including the QSE.

To calculate the former, the classical probability is used instead of the quantum probability density to calculate the density distribution in the absence of QSE. Thus, a continuous approximation for the energy eigenvalues is used, i.e., $\tilde{\varepsilon} \rightarrow \tilde{\varepsilon}_{0}+\tilde{U}(\tilde{x})=(\alpha i)^{2}+\tilde{U}(\tilde{x})$ where $i$ is a continuous quantity running from zero to infinity. Integration over $i$ is used to calculate the summation, so that Eqs. (3) and (4) are rewritten as follows, respectively;

$$
\begin{aligned}
\left(\begin{array}{l}
\mathrm{FD} \\
\mathrm{BE}
\end{array}\right) n(\tilde{x}) & =\frac{1}{\tilde{L}} \int_{0}^{\infty} \frac{d i}{\exp \left[-\Lambda+(\alpha i)^{2}+\tilde{U}(\tilde{x})\right] \pm 1} \\
& =\mp \frac{\sqrt{\pi}}{2 \alpha \tilde{L}} L i_{1 / 2}\left[\mp \exp \left[\Lambda_{L}(\tilde{x})\right]\right] \\
\left(\begin{array}{l}
\mathrm{FD} \\
\mathrm{BE}
\end{array}\right) n_{c l} & =\frac{N}{L}=\frac{1}{\tilde{L}} \int_{0}^{\tilde{L}} n(\tilde{x}) d \tilde{x}= \\
\mp & \frac{\sqrt{\pi}}{2 \alpha \tilde{L}} \int_{0}^{\tilde{L}} L i_{1 / 2}\left[\mp \exp \left[\Lambda_{L}(\tilde{x})\right]\right] d \tilde{x}
\end{aligned}
$$

where $\Lambda_{L}(\tilde{x})=\Lambda-\tilde{U}(\tilde{x})$ and $L i_{1 / 2}$ is the poly-logarithmic function.

The dimensionless density distribution in the absence of QSE is thus,

$$
\left(\begin{array}{l}
\mathrm{FD} \\
\mathrm{BE}
\end{array}\right) \tilde{n}(\tilde{x})=\frac{n(\tilde{x})}{n_{c l}}=\frac{L i_{1 / 2}\left[\mp \exp \left[\Lambda_{L}(\tilde{x})\right]\right]}{\int_{0}^{1} L i_{1 / 2}\left[\mp \exp \left[\Lambda_{L}(\tilde{x})\right]\right] d \tilde{x}}
$$

In the case of Bose gas, $\Lambda_{L}(\tilde{x})=\Lambda-\tilde{U}(\tilde{x})<0$, and therefore, the upper limit for the zero-dimensional global chemical potential is of the form $\Lambda<-1 / \tilde{T}$. In the Maxwell-Boltzmann (MB) limit, Eq. (9) simplifies to,

$$
\text { (MB) } \tilde{n}(\tilde{x})=\frac{\exp \left[\Lambda_{L}(\tilde{x})\right]}{\int_{0}^{1} \exp \left[\Lambda_{L}(\tilde{x})\right] d \tilde{x}}=\frac{\exp [-\tilde{U}(\tilde{x})]}{\int_{0}^{1} \exp [-\tilde{U}(\tilde{x})] d \tilde{x}}
$$

\subsection{Investigation of the density distribution for classical and quantum gases}

The dimensionless local density distributions of a ${ }_{2} \mathrm{He}^{4}$ gas confined in a one-dimensional domain made of silicon material, $1 \mathrm{~nm}$ in length, are given in Figures 2, 3 and 4 for the case of MB statistics for different temperatures. The red curves in the figures show the density distribution with QSE calculated according to Eq. (5) and the blue curves show the densities without QSE calculated according to Eq. (10).

As can be seen in Figures 2, 3 and 4, the contribution of QSE to the density distribution is negligible at high temperatures, while at low temperatures it causes a considerable difference. Both densities with and without QSE decrease to zero rapidly at the limit $\tilde{r}_{0}$. Thus, there is a boundary layer due to the repulsive part of the $\mathrm{LJ}$ potential. This layer may be called the LJ boundary layer, and it is about $\tilde{r}_{0}$ in thickness. In addition, the negative peaks of the LJ potential lead to the LJ peaks in density. However, it is seen that total boundary layer becomes thicker due to the presence of QSE. The wave character of the particles drives the gas towards the inner regions of the domain and, therefore, the $\mathrm{LJ}$ peaks decrease.

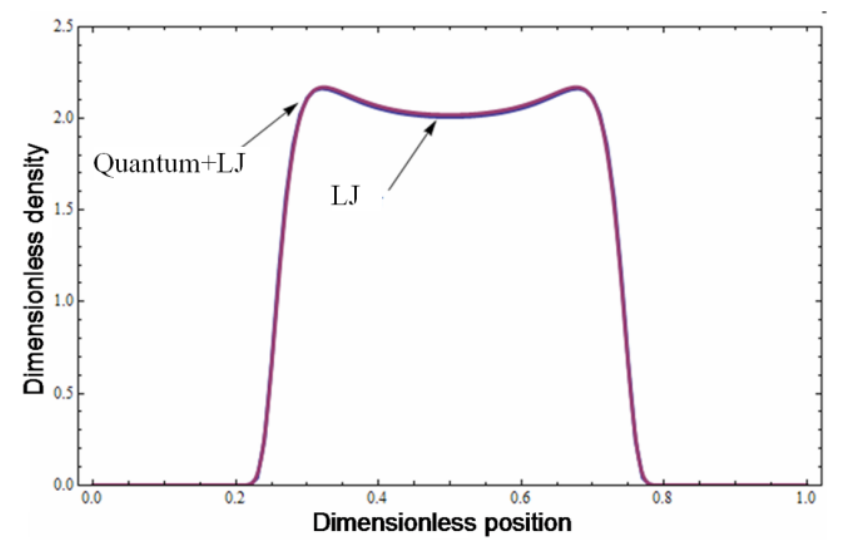

Figure 2. The dimensionless local density distribution of a Maxwellian gas in a one-dimensional domain with, $\alpha=0.045$ and $T=300 K$ (figure is in color in the on-line version of the paper).

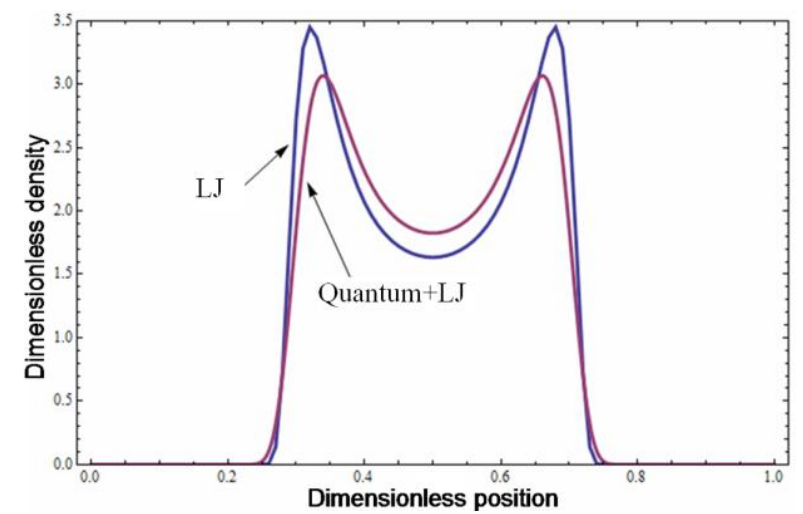

Figure 3. The dimensionless local density distribution of a Maxwellian gas in a one-dimensional domain with $\alpha=0.14$ and $T=30 \mathrm{~K}$ (figure is in color in the on-line version of the paper).

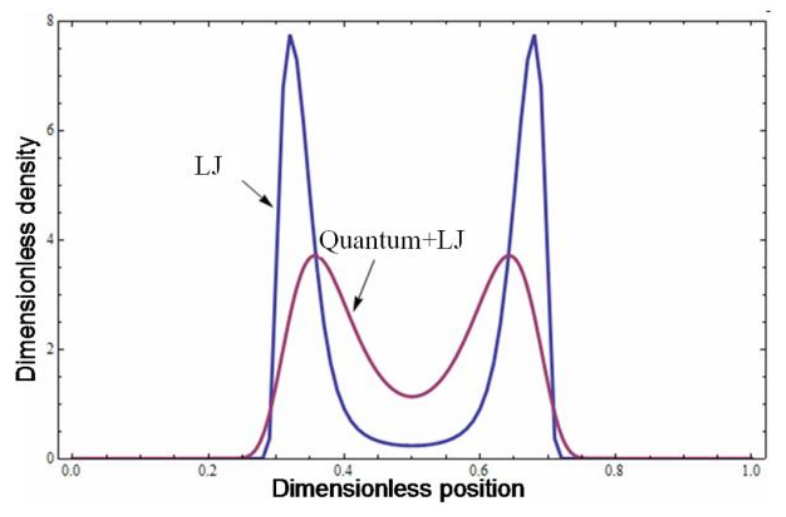

Figure 4. The dimensionless local density distribution of a Maxwellian gas in a one-dimension domain with $\alpha=0.35$ and $T=5 K$ (figure is in color in the on-line version of the paper). 
In Figure 5, the local density distribution of a MB gas confined in a square domain, $\left(1 \mathrm{~nm}^{2}\right)$, subject to $\mathrm{LJ}$ potential is given for $300 \mathrm{~K}$. Because $T_{L J}=30 \mathrm{~K}$, it can be seen that the LJ peaks are weakened at $300 \mathrm{~K}$. In addition, the $2 \mathrm{D}$ distribution is obtained by multiplying the $1 \mathrm{D}$ distribution for each direction given in Figure 2.


Figure 5. The dimensionless local density distribution of a Maxwellian gas in a square domain subject to the LJ potential $\left({ }^{4} \mathrm{He}\right.$ and $\mathrm{Si}$ wall interaction), with $\alpha=0.045$ and $T=300 \mathrm{~K}$ (figure is in color in the on-line version of the paper).

In Figures 6, 7 and 8, the local density distribution of a degenerate Fermi gas is given at different temperatures.



Figure 6. The dimensionless local density distribution of a Fermi gas in a one-dimension subject to the LJ potential $\left({ }^{3} \mathrm{He}\right.$ and $\mathrm{Si}$ wall interaction), with $\alpha=0.045, T=300 \mathrm{~K}$, and $\Lambda=5$ (figure is in color in the on-line version of the paper).

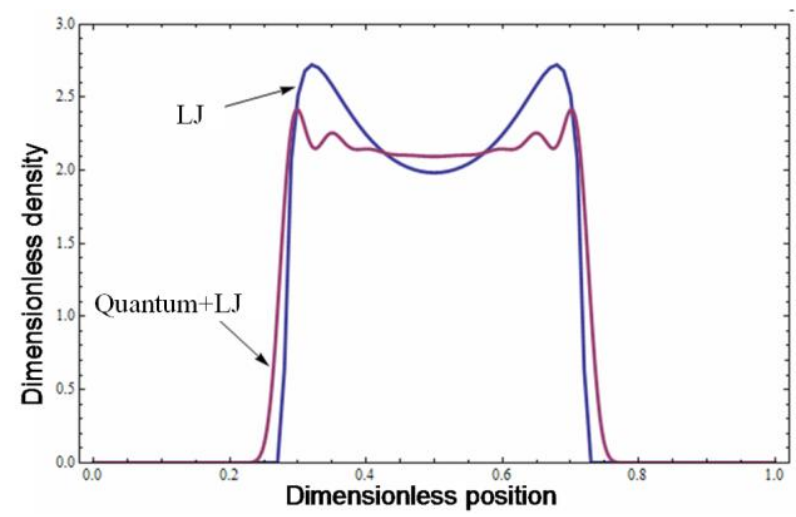

Figure 7. The dimensionless local density distribution of a Fermi gas in a one-dimension domain subject to the LJ potential $\left({ }^{3} \mathrm{He}\right.$ and $\mathrm{Si}$ wall interaction) with $\alpha=0.14, T=30$ $K$, and $\Lambda=5$ (figure is in color in the on-line version of the paper).

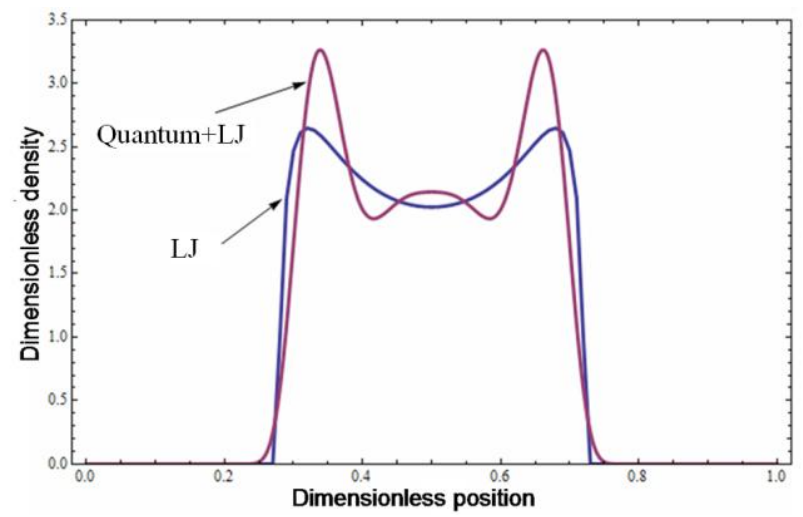

Figure 8. The dimensionless local density distribution of a Fermi gas in a one-dmension domain subject to the $L J$ potential $\left({ }^{3} \mathrm{He}\right.$ and $\mathrm{Si}$ wall interaction) with $\alpha=0.35, T=5$ $K$, and $\Lambda=5$ (figure is in color in the on-line version of the paper).

In Figures 9 and 10, the local density distribution, with QSE of a degenerate Fermi gas confined in a square domain subject to the LJ potential is given for $30 \mathrm{~K}$ and $5 \mathrm{~K}$, respectively. It is clearly seen that the Friedel oscillations are dominant at $5 \mathrm{~K}$, that the LJ peaks still exist, and that large peaks occur in the distribution due to overlapping with the first peak of Friedel oscillations and the LJ peak [Tüttő \& Zawadowski, 1985].

In Figures 11, 12 and 13, the local density distributions of a degenerate Bose gas are given at different temperatures. In a Bose gas, the LJ peaks seem to be stronger in the absence of QSE even at $300 \mathrm{~K}$ relative to those with QSE and become even more so as the temperature decreases. The reason for this is the tendency of Bose particles to be in the same quantum state with the lowest energy. In addition, the QSE disturb the particle distribution by making the midpoint of the domain as a preferred region. Since the total particle number is conserved, the LJ peaks are weakened.

In Figures 14 and 15 the local density distribution, with QSE, of a degenerate Bose gas confined in a square domain subject to the $\mathrm{LJ}$ potential is given for $30 \mathrm{~K}$ and $5 \mathrm{~K}$, respectively. It is observed that the $\mathrm{LJ}$ peaks continue to be the preferred region in the case of the Bose gas. It is clearly seen that QSE cause the distribution to be much smoother and force the particles to be accumulated the inner region of the domain. 

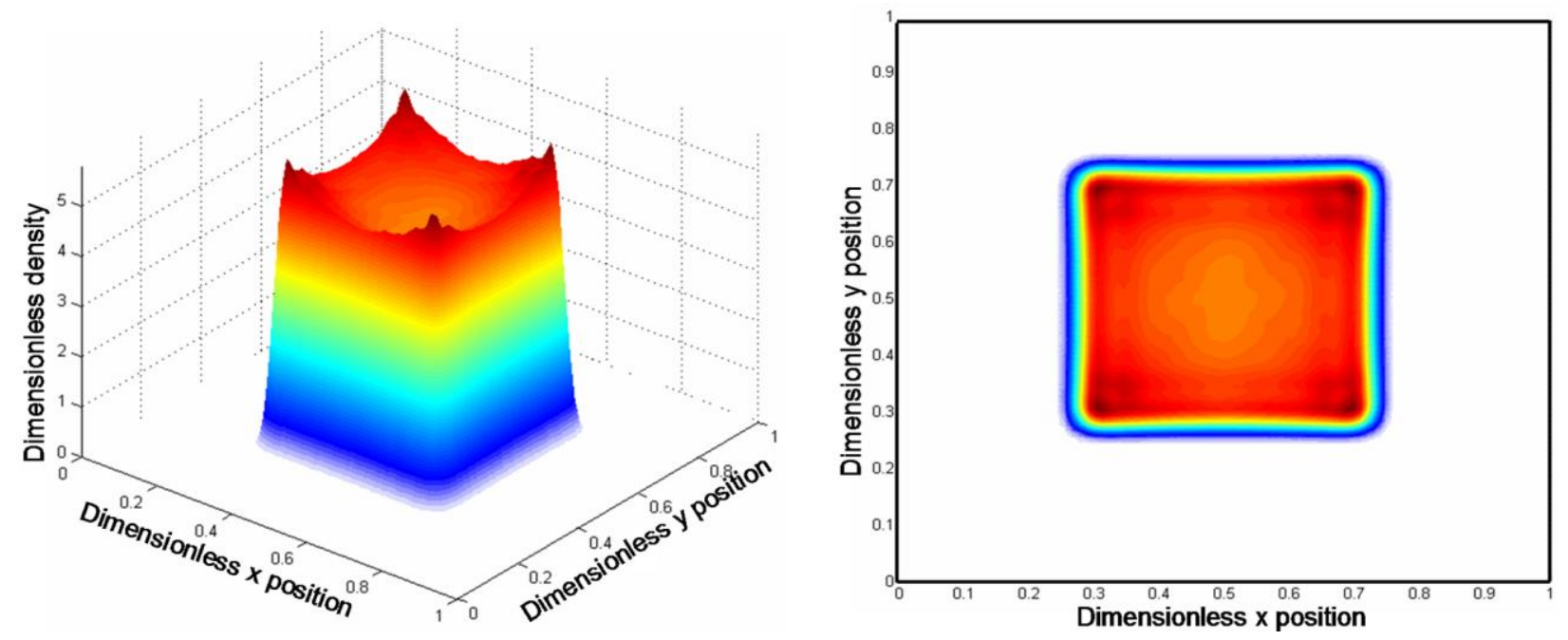

Figure 9. The dimensionless local density distribution of a Fermi gas in a square-shaped domain subject to the LJ potential ( ${ }^{3} \mathrm{He}$ and Si wall interaction) with $\alpha=0.14, T=30 \mathrm{~K}$, and $\Lambda=5$ (figure is in color in the on-line version of the paper).


Figure 10. The dimensionless local density distribution of a Fermi gas in a square-shaped domain subject to the LJ potential $\left({ }^{3} \mathrm{He}\right.$ and Si wall interaction) with $\alpha=0.35, T=5 \mathrm{~K}$, and $\Lambda=5$ (figure is in color in the on-line version of the paper).

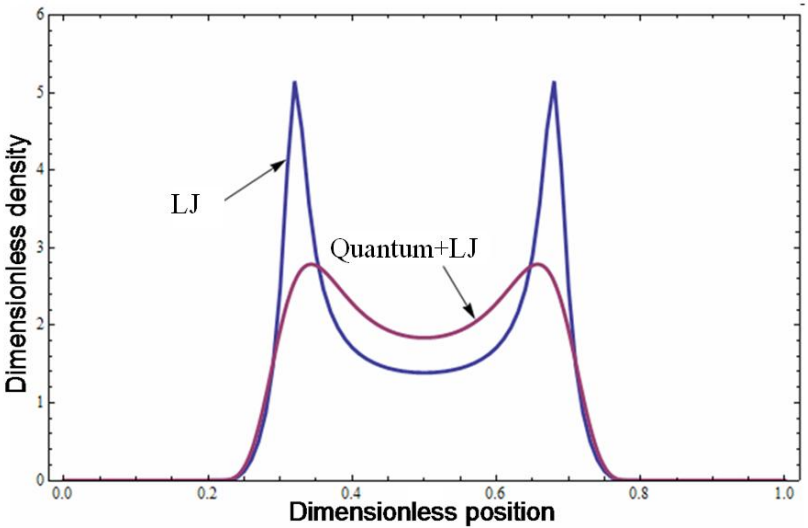

Figure 11. The dimensionless local density distribution of a Bose gas in a 1D domain subject to the LJ potential (He4 and $\mathrm{Si}$ wall interaction) with $\alpha=0.045, T=300 \mathrm{~K}$, and $\Lambda=-$ 0.11 (figure is in color in the on-line version of the paper).

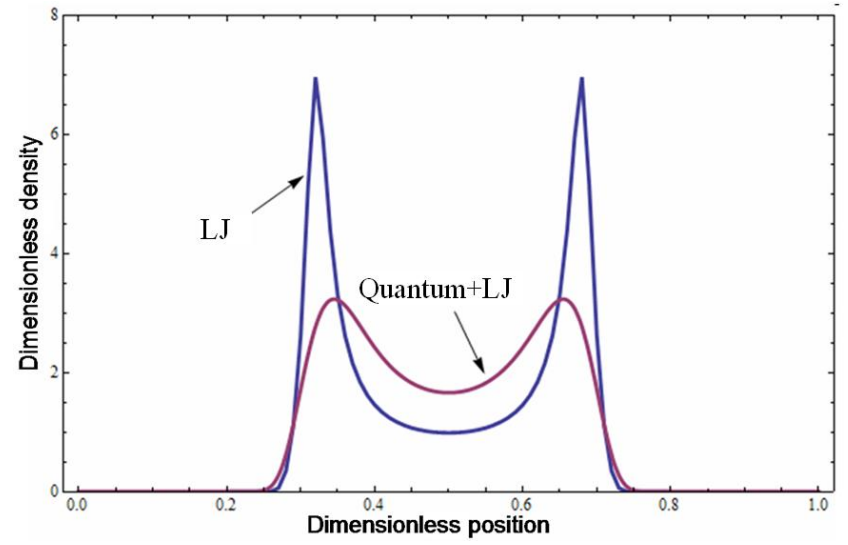

Figure 12. The dimensionless local density distribution of a Bose gas in a $1 D$ domain subject to the LJ potential $\left({ }^{4} \mathrm{He}\right.$ and Si wall interaction) with $\alpha=0.14, T=30 \mathrm{~K}$, and $\Lambda=-1.11$ (figure is in color in the on-line version of the paper). 




Figure 13. The dimensionless local density distribution of a Bose gas in a $1 D$ domain subject to the LJ potential $\left({ }^{4} \mathrm{He}\right.$ and $\mathrm{Si}$ wall interaction) with $\alpha=0.35, T=5 \mathrm{~K}$, and $\Lambda=-6.14$ (figure is in color in the on-line version of the paper).


Figure 14. The dimensionless local density distribution of a Bose gas in a square-shaped domain subject to the LJ potential with $\alpha=0.14, T=30 \mathrm{~K}$, and $\Lambda=-1.11$ (figure is in color in the on-line version of the paper).
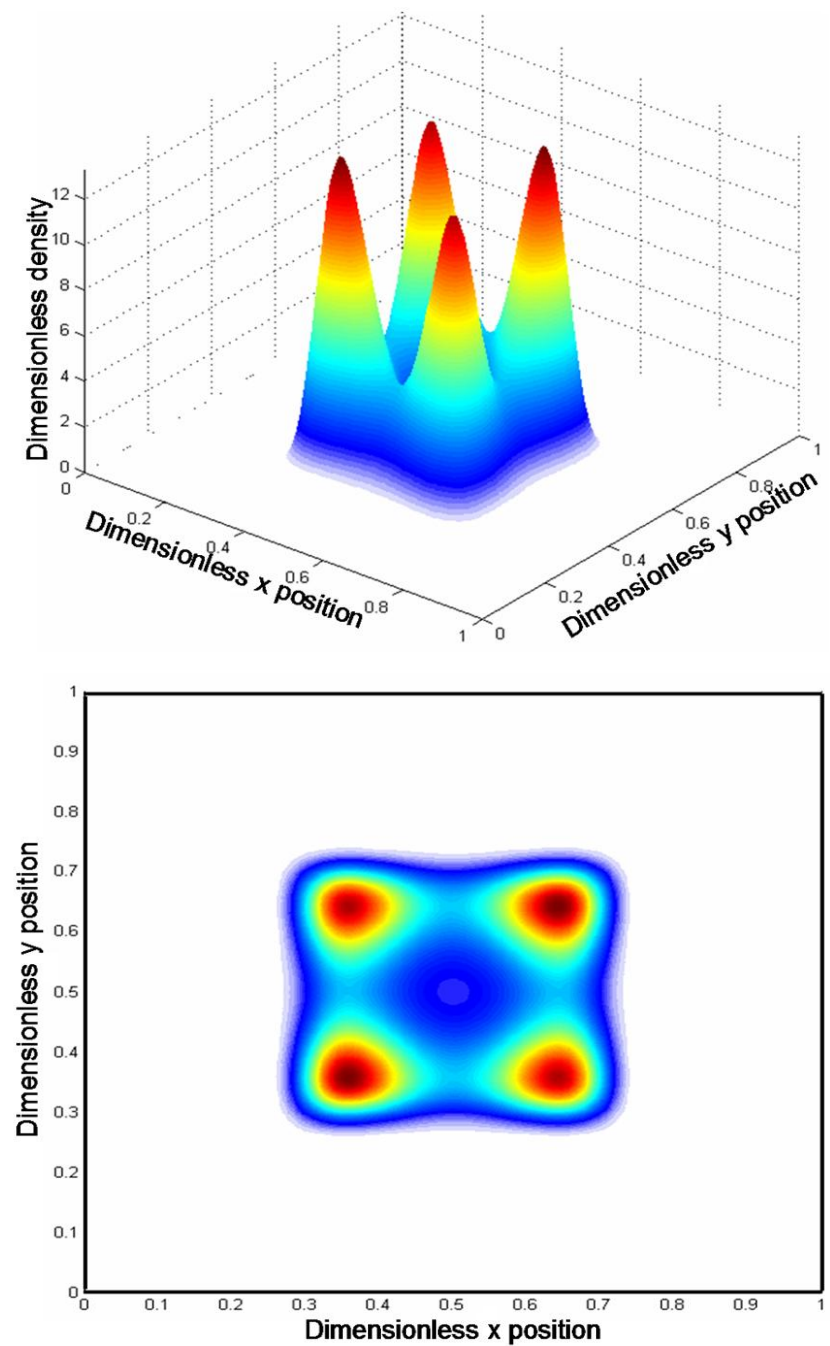

Figure 15. The dimensionless local density distribution of a Bose gas in a square-shaped domain subject to the LJ potential $\left({ }^{4} \mathrm{He}\right.$ and $\mathrm{Si}$ wall interaction) with $\alpha=0.35, T=5 \mathrm{~K}$, and $\Lambda=-6.14$ (figure is in color in the on-line version of the paper).

\section{Conclusion}

In this study, the local density distribution in 1D and 2D for both classical (MB) and quantum (FD and $\mathrm{BE}$ ) gases subject to a $\mathrm{LJ}$ (12-6) interaction potential are examined by considering the existence of QSE. Both density distributions with and without QSE decrease to zero rapidly before the limit $\tilde{r}_{0}$. Thus, there is a boundary layer with thickness $\tilde{r}_{0}$ due to the LJ potential which is called here the $\mathrm{LJ}$ boundary layer. In addition, the negative peaks of the $\mathrm{LJ}$ potential lead to the LJ peaks in density. However, it is seen that the boundary layer observed in density becomes thicker due to the existence of QSE and drives the gas towards the inner regions of the domain with the result that the $\mathrm{LJ}$ peaks reduce.

All of the density-dependent thermodynamic properties are thus, affected by the inhomogeneity in the density distribution. Therefore, it is important to consider these effects on local density to analyze the thermodynamic behaviors of gases confined in nano structures. A detailed analysis of these effects on local density also provides a basis for the experimental verification of QSE on local density due to the wave character of particles. 


\section{Acknowledgements}

This work is supported by The Scientific and Technological Research Council of Turkey, TUBITAK, under contract number $105 T 086$ and the Istanbul Technical University Scientific Research Program.

\section{Nomenclature}

$A \quad$ Surface area of the domain $\left(\mathrm{nm}^{2}\right)$

$i \quad$ continuous quantity running from zero to infinity

$k_{b} \quad$ Boltzmann's constant $\left(1.38 \times 10^{-23} \mathrm{~J} / \mathrm{K}\right)$

$L \quad$ domain size (nm)

$L_{c} \quad$ half of the most probable de Broglie wavelength (nm)

$\mathrm{Li} \quad$ poli-logarithmic function

$n \quad$ particle number density $\left(\# / \mathrm{nm}^{3}\right)$

$N \quad$ total number of particles

$r_{0} \quad$ distance at which the inter-particle potential is zero $(\mathrm{nm})$

$T \quad$ temperature (K)

$U \quad$ energy $(\mathrm{kJ} / \mathrm{mol})$

$V \quad$ Volume of the domain $\left(\mathrm{nm}^{3}\right)$

$x \quad$ distance between particles $(\mathrm{nm})$

\section{Greek Symbols}

$\alpha \quad$ dimensionless $L_{c}$

$\varepsilon \quad$ energy eigenvalue (J) or depth of the potential well $(\mathrm{kJ} / \mathrm{mol})$

$\Lambda \quad$ dimensionless chemical potential

$\Lambda_{L} \quad$ dimensionless local chemical potential

$\lambda$ de Broglie wavelength of particle (nm)

$\psi \quad$ eigenfunction

\section{Sub- and superscripts}

$\sim \quad$ dimensionless property

cl Classical

LJ Lennard-Jones

$r \quad$ quantum state

$T \quad$ thermal

$\begin{array}{ll}\text { Abbreviations } \\ \text { BE } & \text { Bose-Einstein } \\ \text { FD } & \text { Fermi-Dirac } \\ \text { LJ } & \text { Lennard-Jones } \\ \text { MB } & \text { Maxwell-Boltzmann } \\ \text { QSE } & \text { quantum size effects }\end{array}$

\section{References}

Kang, J. W., Hwang, H. J., Lee, J.H. and Lee, H. J. (2004). Fluidic gas-driven carbon-nanotube motor: Molecular dynamics simulations, J. Korean Phys. Society, 45, 573576.

Kang, J. W., and Hwang, H. J. (2004). Nanoscale carbon nanotube motor schematics and simulations for micro electro-mechanical machines, Nanotechnology, 15, 1633-1638.

Moriarty, P. (2001). Nanostructured materials, Rep. Prog. Phys. 64, 297-381.

Terrones, M., Kamalakaran, R., Seeger, T. and Rühle, M. (2000). Novel nanoscale gas containers: encapsulation of N2 in CNx nanotubes, Chem. Commun, 2335-2336.
Cumings, J. and Zettl, A. (2000), Low-Friction Nanoscale Linear Bearing Realized from Multiwall Carbon Nanotubes, Science, 289, 602-604.

Hoummady, M. and Fujita, H. (1999). Micromachines for nanoscale science and technology, Nanotechnology, 10, 29-33.

Lijima, S. and Ichihashi, T. (1993). Single-shell carbon nanotubes of 1-nm diameter, Nature, 363, 603-605.

Lijima, S. (1991), Helical Microtubules of graphitic carbon, Nature, 354, 56-58.

Molina, M. I. (1996). Ideal gas in a finite container, Am.J.Phys., 64, 503-505.

Gutierrez, G. and Yanez, J. M. (1997). Can an ideal gas feel the shape of its container?, Am. J. Phys., 65, 739-743.

Pathria, R. K. (1998). An ideal quantum gas in a finitesized container, Am. J. Phys., 66, 1080-1085.

Dai W.S. and Xie M. (2003), Quantum statistics of ideal gases in confined space, Phys. Lett. A, 311, 340-346.

Sisman, A. and Müller, I. (2004). The Casimir-like size effects in ideal gases, Phys. Lett._A, 320, 360-366.

Dai W.S. and Xie, M. (2004). Geometry effects in confined space, Phys. Rev. E, 70, 016103-1.

Sisman, A. (2004). Surface dependency in thermodynamics of ideal gases, J.Phys. A: Math. Gen., 37, 11353-11361.

Pang, H., Dai,W.S. and Xie, M. (2006). The difference of boundary effects between Bose and fermi systems, $J$. Phys. A: Math. Gen., 39, 2563-2571.

Sisman, A., Ozturk, Z.F., and Firat, C. (2007), "Quantum boundary layer: a non-uniform density distribution of an ideal gas in thermodynamic equilibrium", Phys. Lett. A, 362 , pp.16-20.

Firat, C., Sisman, A., and Ozturk, Z.F. (2010). "Thermodynamics of gases in nano cavities", Energy, $35,814-819$.

Firat, C., Sisman, A. (2009). "Universality of quantum boundary layer for a Maxwellian gas ”, Physica Scripta, 79:6, 065002(1-5).

Hirschfelder, J. O., Curtiss, C. F. and Bird, R. B. (1954). Molecular Theory of Gases and Liquids, John Wiley \& Sons, New York.

Israelachvili, J. N. (1992). Intermolecular and Surface Forces, Academic Press, London.

Nagy, L. T., Tunega, D. and Liska, M. (1996), Modeling of interaction properties of surfaces of phyllosilicates: A theoretical forecast of adsorption isotherms of noble gases at the talc surface, Int. J. Quantum Chem., 57, 843-849.

Griffiths, D. J. (1995). Introduction to Quantum Mechanics, Prentice Hall, New Jersey.

Tüttő, I. and Zawadowski, A. (1985). Quantum theory of local perturbation of the charge-density wave by an impurity: Friedel oscillations, Phys.Rev.B, 32, 24492470. 